\section{Mutation signature of aristolochic acid}

Aristolochic acid is produced in Aristolochia plants, which are components of herbal medicines commonly used in Asia. Aristolochic acid was classified as a carcinogen after exposure was associated with upper urinary tract urothelial carcinoma (UTUC). Now, two groups separately determine the mutational signature of aristolochic acid. Bin Teah Teh, Patrick Tan, Steven Rozen, See-Tong Pang and colleagues report whole-genome sequencing of one aristolochic acid-exposed UTUC tumor and exome sequencing of eight additional exposed tumors (Sci. Transl. Med. 5, 197ra101, 2013). Separately, Thomas Rosenquist, Kenneth Kinzler and colleagues report exome sequencing of 19 aristolochic acid-exposed UTUCs and 7 non-exposed UTUCs (Sci. TransI. Med. 5,197 ra 102, 2013). Both groups observed a very high mutation rate in exposed tumors and identified recurrent somatic mutations in genes that encode chromatin modifiers. They both determined that the aristolochic acid mutation signature is characterized by A:T to $\mathrm{T}: \mathrm{A}$ transversions that preferentially occur at a specific sequence motif. This sequence motif occurs in the canonical splice acceptor site, and both groups found an over-representation of splice-site mutations. These papers shed light on the mutagenic properties of this carcinogen in herbal remedies.

\section{Schizophrenia exomes}

A new study from Mary-Claire King and colleagues reports exome sequencing in sporadic schizophrenia (Cell 154, 518-529, 2013). The goal of the study design was to enrich for de novo mutations by focusing on patients with sporadic (not familial) disease. The authors report exomes from 105 patients, 84 unaffected siblings and 210 unaffected parents. Forty-seven of the 105 probands (45\%) carried de novo mutations predicted to damage protein function, whereas 25 of the 84 unaffected siblings (30\%) harbored 35 such mutations. These results indicate a modest enrichment within probands of a higher likelihood of harboring at least one de novo damaging mutation $(P=0.035)$. The authors list the genes that carry damaging de novo mutations in patients, but none appear to be recurrently mutated. Further resequencing of these new candidates in large cohorts may be useful to prove causality. The authors performed transcriptional network analyses using RNA sequencing data from the BrainSpan Atlas and found that in fetal frontal cortex the networks of genes with de novo damaging mutations in patients showed greater connectedness in coexpression than networks derived from genes with de novo mutations in controls. This result offers support for the hypothesis that disruptions in fetal prefrontal cortical development are a key pathogenic mechanism in schizophrenia.

PF

\section{Hexokinase 2 required in tumors}

It is well known that cancer cells have elevated glucose metabolism, and much effort has been directed at identifying targets that will selectively disrupt this pathway in cancer. Hexokinases catalyze the first committed step in glucose metabolism and are highly expressed in cancer cells. Using Hk2 conditional knockout mice, Nissim Hay and colleagues show that hexokinase 2 is required for tumor initiation and maintenance in mouse models of cancer (Cancer Cell doi:10.1016/ j.ccr.2013.06.014, 1 August 2013). The authors crossed mice carrying activated KRas (Kras $\left.{ }^{L S L-G 12 D}\right)$, a model of non-small-cell lung cancer,

Written by Orli Bahcall, Pamela Feliciano \& Emily Niemitz or mice carrying activated ErbB2 (Neu), a model of breast cancer, to $H k 2$ conditional knockout mice. They found that the average tumor size in $\mathrm{Kras}^{L S L-G 12 D}$; $\mathrm{Hk}^{-/-}$mice was significantly smaller than in $K r a s^{L S L-G 12 D} ; H k 2^{f l f l}$ undeleted controls. They also found that mortality was profoundly delayed in the mice with activated KRas and $\mathrm{Hk}^{-/-}$. Loss of $H k 2$ had a similar effect in ErbB2-driven mammary gland tumorigenesis, as $\mathrm{Hk} 2$ deletion slowed the onset of tumors and reduced their incidence. Small-molecule inhibitors of HK2 may be a therapeutic approach in cancer, although the authors note that it may be challenging to develop specific inhibitors to HK2 that do not affect the closely related HK1 protein.

$P F$

\section{Transcription factors in combination}

Barak Cohen and colleagues have developed a massively parallel synthetic promoter assay that they use to examine the effects of an array of transcription factor binding site variants (Genome Res. doi:10.1101/ gr.157891.113, 6 August 2013). They used a modified cis-regulatory element sequencing approach that allows for chromosomal integration of large numbers of synthetic promoters in Saccharomyces cerevisiae. They constructed a barcoded reporter library that includes synthetic promoters with a combination of 12 transcription factor binding sites, for a total of 7,289 barcoded sequences representing 2,534 unique cisregulatory elements. Three variants were included for each of four transcription factors-Mig1, Reb1, Rap1 and Gcr1. To examine a wide interaction space, the variants were selected to range from very low to very high predicted binding affinity. To analyze this data set, the authors used a thermodynamic framework that models both transcription factor binding to DNA and interactions between transcription factors. They found that their model replicated known transcription factor relationships and was able to explain $57 \%$ of the variance in expression in the promoter library. They also compared their thermodynamic model predictions to position weight matrix models for binding affinities based on analysis of data from chromatin immunoprecipitation and sequencing (ChIP-seq).

\section{A deeper look back at $Y$}

Two new studies use large-scale sequencing of the $Y$ chromosome to infer key evolutionary dates. Carlos Bustamante and colleagues sequenced the complete $Y$ chromosome of 69 males from 9 diverse populations (Science 341, 562-565, 2013). They identified 11,640 single-nucleotide variants (SNVs), which they used to construct a maximum-likelihood tree. Using archaeological dating for calibration, the authors estimated a mutation rate of $0.82 \times 10^{-9}$ mutations per base pair per year and a Y-chromosome time to most recent common ancestor ( $\left.t_{\text {MRCA }}\right)$ of 120,000-156,000 years ago. The authors also sequenced mitochondrial DNA from the 69 males as well as from 24 females; using a similar analysis, they estimated the mitochondrial DNA $t_{\text {MRCA }}$ at 99,000-148,000 years ago. In a second study, Francesco Cucca and colleagues report low-pass sequencing of the malespecific portion of the $Y$ chromosome (MSY) in 1,204 males from Sardinia (Science 341, 565-569, 2013). They inferred 11,763 phylogenetically informative SNPs and used these to construct a phylogenetic tree. They calibrated against archaeological records, estimating a phylogenetic rate of $0.53 \times 10^{-9}$ mutations per base pair per year and a Y-chromosome $t_{\text {MRCA }}$ of $\sim 180,000-200,000$ years ago. Together, these studies suggest an earlier Y-chromosome coalescence time than previous studies and suggest that this coalescence time may overlap with that for mitochondrial DNA. OB 